Measurement of neutral gas pressure in the D-module of GAMMA 10/PDX by using ASDEX type fast ionization gauge

K. Ichimura, M. Fukumoto, M. M. Islam, M. S. Islam, K. Shimizu, K. Fukui, M. Ohuchi, K. Nojiri, A. Terakado, M. Yoshikawa, N. Ezumi, M. Sakamoto, and Y. Nakashima

Citation: Rev. Sci. Instrum. 87, 11D424 (2016); doi: 10.1063/1.4960536

View online: http://dx.doi.org/10.1063/1.4960536

View Table of Contents: http://aip.scitation.org/toc/rsi/87/11

Published by the American Institute of Physics

Ф SHIMADZU Powerful, Multi-functional UV-Vis-NIR and

Excellence in Science FJJis spectrophotometers

Providing the utmost in sensitivity, accuracy and resolution for applications in materials characterization and science

- Photovoltaics

- Polymers

- Coatings

- Ceramics

- Thin films

- Inks

- DNA film structures

- Packaging materials

- Nanotechnology

- Paints

Click here for accurate, cost-effective laboratory solutions
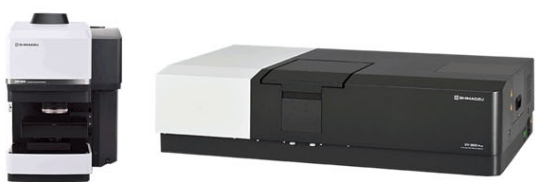


\title{
Measurement of neutral gas pressure in the D-module of GAMMA 10/PDX by using ASDEX type fast ionization gauge
}

\author{
K. Ichimura, ${ }^{1, a)}$ M. Fukumoto, ${ }^{2}$ M. M. Islam, ${ }^{1}$ M. S. Islam, ${ }^{1}$ K. Shimizu, ${ }^{1}$ K. Fukui, ${ }^{1}$ \\ M. Ohuchi, ${ }^{1}$ K. Nojiri, ${ }^{1}$ A. Terakado, ${ }^{1}$ M. Yoshikawa, ${ }^{1}$ N. Ezumi, ${ }^{1}$ M. Sakamoto,,${ }^{1}$ \\ and Y. Nakashima ${ }^{1}$ \\ ${ }^{1}$ Plasma Research Center, University of Tsukuba, 1-1-1 Tennoudai, Tsukuba, Ibaraki, Japan \\ ${ }^{2}$ National Institutes for Quantum and Radiological Science and Technology, 801-1 Mukouyama, \\ Naka, Ibaraki, Japan
}

(Presented 6 June 2016; received 3 June 2016; accepted 18 July 2016;

published online 11 August 2016)

\begin{abstract}
In the divertor simulation experiments in the GAMMA 10/PDX tandem mirror, pressure of the neutral gas was investigated by using a fast ionization gauge. The gauge was absolutely calibrated for hydrogen gas by using a capacitance manometer. Change of the gauge sensitivity due to the magnetic field of GAMMA 10/PDX was also evaluated. The typical gas pressure measured in detached plasma experiments was 0.1-10 Pa. The degree of plasma detachment determined from the reduction of heat flux was enhanced as the gas pressure increases. Rapid increase of the gas pressure under the plasma flow was also observed. Published by AIP Publishing. [http://dx.doi.org/10.1063/1.4960536]
\end{abstract}

\section{INTRODUCTION}

Behavior of a neutral gas in a detached state of divertor plasma is currently a topic of great interest in divertor studies. In a divertor operation, injected neutral gas enhances radiation loss of the plasma, and the heat load from the high temperature plasma is dissipated. For the understanding of the heat mitigation process in the divertor system, behavior of the neutral gas near the target plate is important. The motivation of this research is to observe the behavior of the neutral gas pressure in the D-module (divertor simulation experimental module) of the GAMMA 10/PDX tandem mirror. In GAMMA 10/PDX, ${ }^{1,2}$ detached plasmas are successfully obtained by injecting hydrogen gas and mitigating the heat of end-loss plasma in the D-module. However, the behavior of the gas pressure in a detachment process was unclear. Because the Dmodule is placed in a vacuum and strong magnetic field, a standard ionization gauge is not feasible. Therefore, a diagnostic tool for the gas pressure applicable in a magnetic field is required. A type of fast ionization gauge called ASDEX gauge $^{3,4}$ is able to satisfy the requirement of the gomodule experiments. In addition, the gauge has already been successfully applied in a divertor region of devices such as Doublet III D (DIII-D), ${ }^{5}$ Large Helical Device (LHD) ${ }^{6}$ Compact Helical System (CHS), ${ }^{7}$ and so on. Therefore, the ASDEX type fast ionization gauge was newly installed on the rooftop of the Dmodule. The axis of the gauge was set to be parallel to the axis of the GAMMA 10/PDX. The effect of hydrogen gas pressure for the reduction of heat flux around the $\mathrm{V}$-shaped target plate was investigated using this gauge.

\footnotetext{
Note: Contributed paper, published as part of the Proceedings of the 21st Topical Conference on High-Temperature Plasma Diagnostics, Madison, Wisconsin, USA, June 2016.

${ }^{a)}$ Author to whom correspondence should be addressed. Electronic mail: ichimura@eedept.kobe-u.ac.jp.
}

\section{EXPERIMENTAL DEVICES}

\section{A. GAMMA 10/PDX}

The GAMMA 10/PDX is a large tandem mirror device that has the length of $27 \mathrm{~m}$. The machine consists of several cells; central-cell, anchor-cells, plug/barrier-cells, and end regions. Figure 1 shows the schematic view of the GAMMA 10/PDX vacuum chamber, magnetic coils, and magnetic field lines. In standard experiments, ICRF heating is applied in the anchor-cells and the central-cell. By the heating, hot plasma that typically has $4 \mathrm{keV}$ of ion temperature, $100 \mathrm{eV}$ of electron temperature, and $10^{19} \mathrm{~m}^{-3}$ of electron density is produced. As the nature of a mirror machine, there always exists a loss of plasma particles along the magnetic field lines. Such loss particle is called end-loss particle. The loss particles flow into the end-cells through the open-ended magnetic field. The D-module is installed in the west end-cell and the divertor simulation experiment is performed by channeling the hot endloss plasma to the divertor target mounted in the D-module. Typically, the end-loss plasma has $400 \mathrm{eV}$ of ion temperature, $30 \mathrm{eV}$ of electron temperature, and the particle flux of $5 \times 10^{22}$ ions $\mathrm{m}^{-2} \mathrm{~s}^{-1}$.

\section{B. D-module}

Figure 2 shows the image of the D-module horizontally seen from the north and the schematic diagram of the gas injection systems. The D-module consists of the rectangular box, V-shaped tungsten target, calorimeter, and gas injection systems. The angle of the V-shaped target is able to be changed from $15^{\circ}$ to $80^{\circ}$. The rectangular box is enclosing the $\mathrm{V}$-shaped target in order to achieve high local gas pressure around the target plate. Two gas lines, the upper gas line and the corner gas line, are used to inject neutral gas into the D-module. The gas lines are equipped with valves and reservoir tanks. The flow 


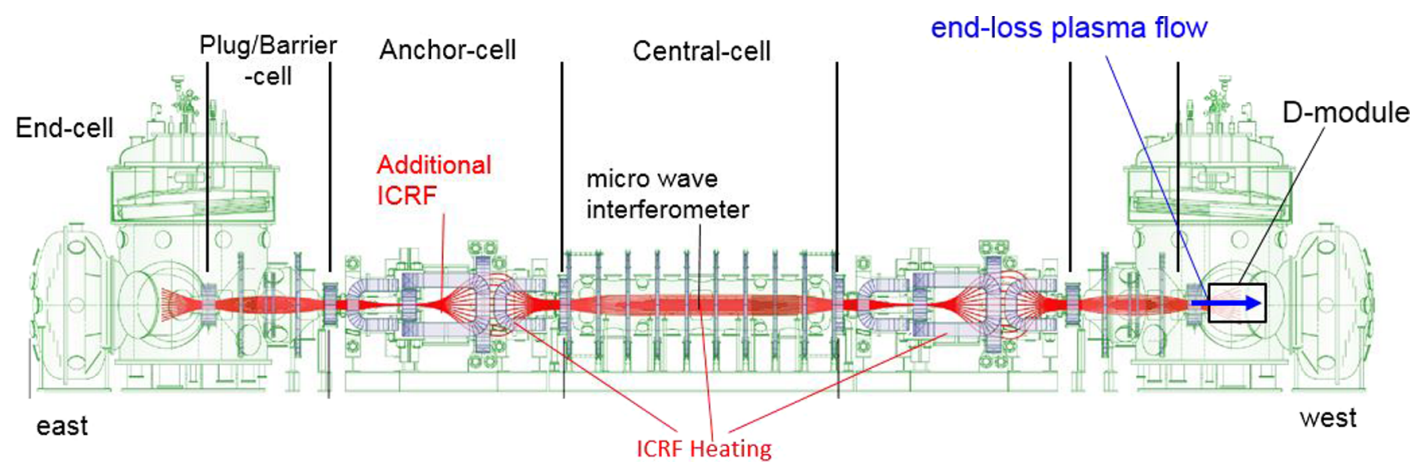

FIG. 1. Schematic view of the vacuum vessels, magnetic coils, and magnetic field lines in GAMMA 10/PDX.

rate of the gas is controlled by changing the plenum pressure in the reservoir tank. Due to the large size of the vacuum vessel of the GAMMA 10/PDX, several meters of tube length is required from the valves to the D-module. As a result, it usually takes $50-100 \mathrm{~ms}$ for hydrogen gas to travel from the valve to the D-module. Therefore in the experiments, valve was opened $300 \mathrm{~ms}$ before the plasma discharge trigger. The calorimeter mounted at the corner of V-shaped target is used to measure the heat flux from the plasma. The ASDEX gauge is mounted in Tshaped tube of ICF70 mounted on the rooftop of the D-module.

\section{ASDEX TYPE FAST IONIZATION GAUGE}

\section{A. Operating principle of the gauge}

The schematic diagram of the ASDEX gauge and the spatial distribution of the potential along the axis of the gauge are shown in Figs. 2(a) and 2(b), respectively. The gauge has the filament, control grid, acceleration grid, and ion collector. First, the filament is heated by the circuit current to emit thermal electron. The electrons are accelerated by the potential of the control grid and acceleration grid. The amount of the thermal electron is monitored as emission current $I_{\mathrm{e}}$ at the acceleration grid. When a neutral particle is ionized by the thermal electron, the ion is accelerated and collected by the ion collector grid. By observing the ion current at the ion collector $\left(I_{\text {ic }}\right)$ and the emission current, amount of neutral gas particle

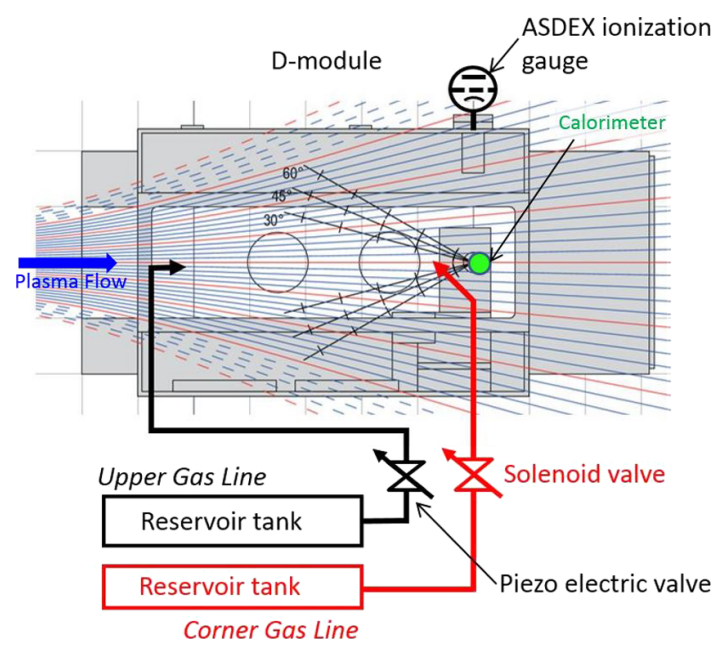

FIG. 2. Image of D-module and the schematic diagram of the gas injection systems. The position of the ASDEX ionization gauge is also indicated. around the gauge can be evaluated. All grids and the filament are aligned in one straight axis. By setting the axis of the gauge along magnetic field line with an acceptance angle of $\pm 25^{\circ}$, the gauge can avoid disturbance from the magnetic field and can keep the linearity of the gauge sensitivity. The gauge can be operated in the range of gas pressure in detached divertor plasma, 0.1-20 Pa (Fig. 3).

\section{B. Calibration of the gauge}

Similar to other ionization gauges, the sensitivity of the ASDEX type ionization gauge changes with the species of the gas. Therefore the calibration of the gauge sensitivity is required for the absolute measurement. The calibration for hydrogen gas was performed in a small vacuum chamber equipped with mass flow controller system, turbo molecular pump, and capacitance manometer. The ASDEX gauge was installed to the small chamber and the value of $I_{\mathrm{ic}}$ and $I_{\mathrm{e}}$ was recorded together with the absolute gas pressure indicated by the capacitance manometer. The result of the calibration experiment is plotted in Fig. 4. The value of the emission current during the calibration was set as $0.5 \mathrm{~mA}$ and the gas pressure in the chamber was controlled by the mass flow controller. The ion collector current $I_{\text {ic }}$ increased almost linearly as the gas pressure increases. Considering a small ambiguity of the linearity, the relation of the ion current, emission current, and the gas pressure was determined as follows:

$$
P_{H_{2}}=615.5\left(\frac{I_{i c}}{I_{e}-I_{i c}}\right)^{2}+95.0\left(\frac{I_{i c}}{I_{e}-I_{i c}}\right) .
$$

It is known that the sensitivity of ASDEX gauge in a magnetic field rises by about an order of magnitude between

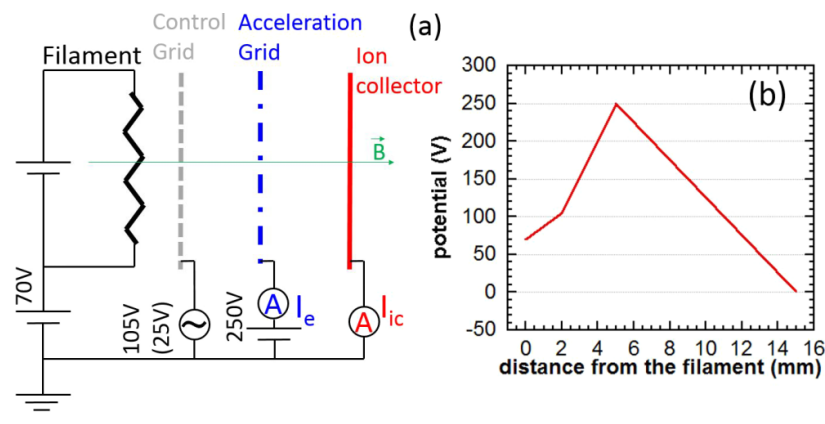

FIG. 3. (a): Schematic diagram of the ionization gauge and (b): spatial distribution of electrostatic potential along the axis of the gauge. 


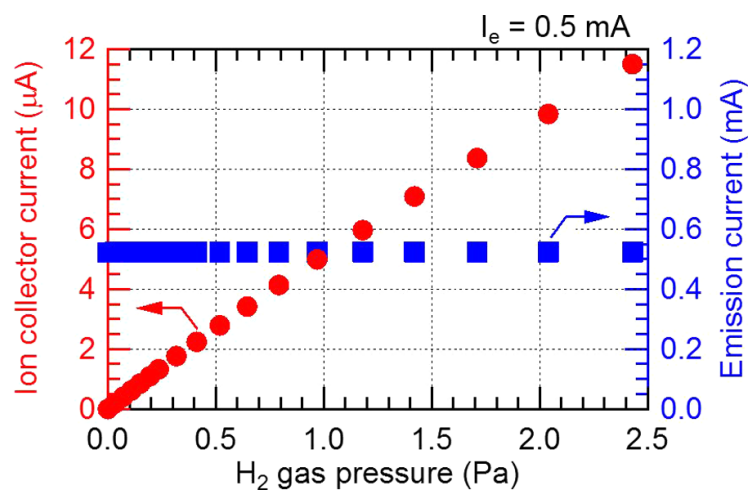

FIG. 4. Measured ion current and emission current plotted against the hydrogen gas pressure monitored by the capacitance manometer.

0 and $1 \mathrm{~T}^{3}$ In GAMMA 10/PDX, the magnetic field at the Dmodule is fixed to the value of about $0.15 \mathrm{~T}$ and has expanding, nozzle-like shape. It was found that the sensitivity of the gauge with the magnetic field is 3.1 times larger than that without the magnetic field. Therefore the equation to evaluate the hydrogen gas pressure in the D-module experiment becomes

$$
P_{H_{2}}=P_{H_{2} \text { with } B} / 3.1 \text {. }
$$

\section{EXPERIMENTAL RESULTS}

Hydrogen gas was injected from the upper gas line in order to investigate the effect of gas pressure on the reduction of the heat load to the target plate. The angle of the V-shaped target is fixed as $45^{\circ}$. Figure 5 is the time evolution of the measured hydrogen gas pressure with the electron line density of the plasma in the central-cell measured by the microwave interferometer. In this operation, hydrogen gas was injected with the plenum pressure of $60 \mathrm{kPa}$. The discharge of the plasma starts from $50 \mathrm{~ms}$, and as the plasma is started, the pressure of the neutral gas rapidly increased. The gas pressure was increased more as the density of the upstream plasma was increased by the additional heating of ICRF. In average, the gas pressure became 3.5 times larger during the plasma discharge. The expected increase of gas pressure due to the particle flux of $5 \times 10^{22}$ ions $\mathrm{m}^{-2} \mathrm{~s}^{-1}$ is less than $0.1 \mathrm{~Pa}$.

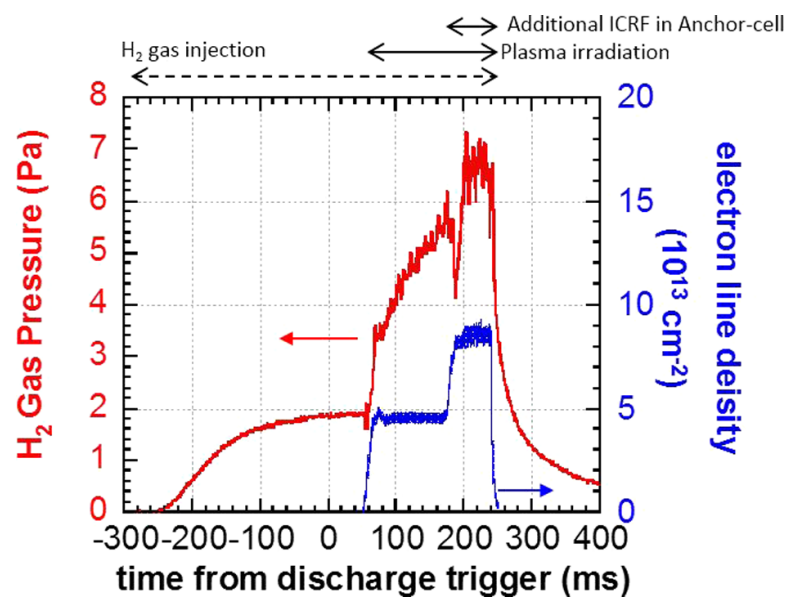

FIG. 5. Time evolution of the hydrogen gas pressure in the D-module and the electron line density of the central-cell plasma.

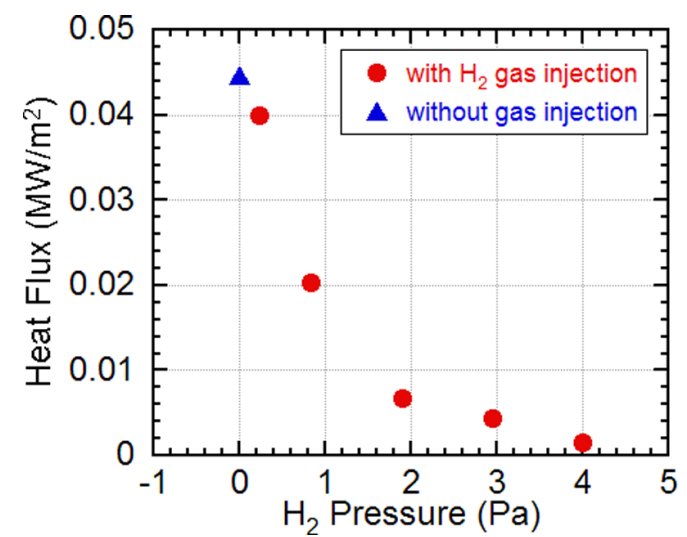

FIG. 6. The heat flux measured at the corner of the V-shaped target plotted against the hydrogen gas pressure at $\mathrm{t}=45 \mathrm{~ms}$.

Therefore, the compression of the neutral gas by the plasma flow is considered to be the cause of the pressure increase.

The reduction of the heat flux was evaluated by the calorimeter mounted at the corner of the $\mathrm{V}$-shaped target. The gas pressure in the $\mathrm{D}$-module was changed by varying the plenum pressure by $20 \mathrm{kPa}$ steps. In Fig. 6, measured heat flux is plotted against the hydrogen pressure in the $\mathrm{D}$-module. The gas pressure indicated in the plot was evaluated at the time slightly before the plasma discharge in order to take the effect of gas compression off from the analysis. It is obvious that the heat load from the plasma flow becomes smaller as the amount of gas increases. About $90 \%$ of the heat flux was removed by the hydrogen gas of $3 \mathrm{~Pa}$.

\section{SUMMARY}

For the measurement of neutral gas pressure in the Dmodule of GAMMA 10/PDX, the ASDEX type ionization gauge was applied. The calibration of the gauge sensitivity for hydrogen gas in the magnetic field of GAMMA 10/PDX was successfully performed. It was found that the gas pressure of $0.1-10 \mathrm{~Pa}$ is obtained by the gas injection. The heat flux guided to the target plate became smaller as the gas pressure increased. The temporal behavior of the gas pressure indicated that the injected gas is compressed in the D-module by the flow of plasma. Details of the gas compression and the gauge sensitivity in different magnetic field strength and different gas species will be analyzed in future.

\section{ACKNOWLEDGMENTS}

This study was supported by the NIFS as bi-directional collaborative research (Grant Nos. NIFS12KUGM066 and NIFS16KUGM117). The authors thank the members of the GAMMA 10 research group for their collaboration on the experiments and for helpful discussions.

\footnotetext{
${ }^{1}$ Y. Nakashima et al., Fusion Eng. Des. 85, 956-962 (2010).

${ }^{2}$ Y. Nakashima et al., Fusion Sci. Technol. 63(1T), 100-105 (2013).

${ }^{3}$ G. Haas et al., J. Nucl. Mater. 121, 151 (1984).

${ }^{4}$ G. Haas and H.-S. Bosch, Vacuum 51, 39-46 (1998).

${ }^{5}$ D. N. Hill et al., Rev. Sci. Instrum. 61, 3548 (1990).

${ }^{6}$ S. Masuzaki et al., J. Nucl. Mater. 363-365, 314-318 (2007).

${ }^{7}$ A. Komori et al., J. Nucl. Mater. 241-243, 967-971 (1997).
} 